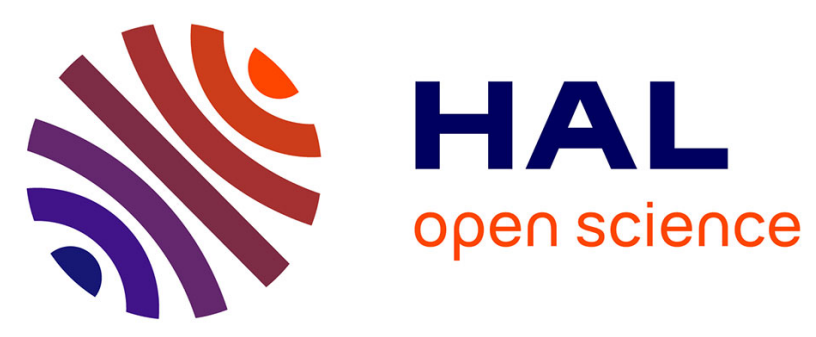

\title{
Risk Factors of Inadequate Colposcopy After Large Loop Excision of the Transformation Zone: A Prospective Cohort Study
}

Xavier Carcopino, Julien Mancini, Jean Gondry, Julien Chevreau, Gery Lamblin, Anthony Atallah, Vincent Lavoué, Claire Caradec, Jean Baldauf, Angélique Bryand, et al.

\section{To cite this version:}

Xavier Carcopino, Julien Mancini, Jean Gondry, Julien Chevreau, Gery Lamblin, et al.. Risk Factors of Inadequate Colposcopy After Large Loop Excision of the Transformation Zone: A Prospective Cohort Study. Journal of Lower Genital Tract Disease, 2018, 22 (1), pp.31-37. 10.1097/LGT.0000000000000357 . hal-02058148

\section{HAL Id: hal-02058148 \\ https://hal-amu.archives-ouvertes.fr/hal-02058148}

Submitted on 23 Apr 2019

HAL is a multi-disciplinary open access archive for the deposit and dissemination of scientific research documents, whether they are published or not. The documents may come from teaching and research institutions in France or abroad, or from public or private research centers.
L'archive ouverte pluridisciplinaire HAL, est destinée au dépôt et à la diffusion de documents scientifiques de niveau recherche, publiés ou non, émanant des établissements d'enseignement et de recherche français ou étrangers, des laboratoires publics ou privés. 


\title{
Risk Factors of Inadequate Colposcopy After Large Loop Excision of the Transformation Zone: A Prospective Cohort Study
}

\author{
Xavier Carcopino, MD, PhD, ${ }^{1}$ Julien Mancini, MD, $P h D,{ }^{2,3}$ Jean Gondry, MD, $P h D,{ }^{4}$ Julien Chevreau, MD, ${ }^{4}$ \\ Gery Lamblin, MD, ${ }^{5}$ Anthony Atallah, $M D,{ }^{5}$ Vincent Lavoue, MD, PhD, ${ }^{6}$ Claire Caradec, $M D,{ }^{6}$ \\ Jean-Jacques Baldauf, MD, PhD, ${ }^{7}$ Angélique Bryand, MD, ${ }^{7}$ Sebastien Henno, MD, ${ }^{8}$ Aubert Agostini, MD, PhD, ${ }^{9}$ \\ Serge Douvier, MD, PhD, ${ }^{10}$ Adelaïde Jarniat, MD, ${ }^{10}$ Didier Riethmuller, MD, PhD, ${ }^{11}$ Anne Mendel, MD, ${ }^{11}$ \\ Jean-Luc Brun, MD, PhD, ${ }^{12}$ Hajanirina Rakotomahenina, $M D,{ }^{12}$ and Lise Preaubert, $M D^{1}$
}

\begin{abstract}
Objective: The aim of the study was to identify the risk factors of post-large loop excision of the transformation zone (LLETZ) inadequate colposcopy. Materials and Methods: From December 2013 to July 2014, a total of 157 patients who had a LLETZ performed for the treatment of high-grade intraepithelial lesion with fully visible cervical squamocolumnar junction were included. All procedures were performed using semicircular loops. The use of colposcopy made during each procedure was systematically documented. Dimensions and volume of LLETZ specimens were measured at the time of procedure, before formaldehyde fixation. All participants were invited for a follow-up colposcopy 3 to 6 months after LLETZ. Primary end point was the diagnosis of post-LLETZ inadequate colposcopy, defined by a not fully visible cervical squamocolumnar junction and/or cervical stenosis.
\end{abstract}

Results: Colposcopies were performed in a mean (SD) delay of 136 (88) days and were inadequate in $22(14 \%)$ cases. Factors found to significantly increase the probability of post-LLETZ inadequate colposcopy were a history of previous excisional cervical therapy [adjusted odds ratio $(\mathrm{aOR})=4.29,95 \% \mathrm{CI}=1.12-16.37, p=.033]$ and the thickness of the specimen $(\mathrm{aOR}=3.12,95 \% \mathrm{CI}=1.02-9.60, p=.047)$. The use of colposcopy for the guidance of LLETZ was statistically associated with a decrease in the risk of post-LLETZ inadequate colposcopy ( $\mathrm{aOR}=0.19,95 \%$

${ }^{1}$ Department of Obstetrics and Gynaecology, Hôpital Nord, APHM, AixMarseille University (AMU), Univ Avignon, CNRS, IRD, IMBE UMR 7263, Marseille, France; ${ }^{2}$ Aix Marseille University, Inserm, IRD, Marseille, France; ${ }^{3}$ APHM, Hôpital de la Timone, Public Health Department, BioSTIC, Marseille, France; ${ }^{4}$ University Hospital of Amiens, Jules Verne University, Picardie, France; ${ }^{5}$ Department of Obstetrics and Gynaecology, Femme Mère Enfant University Hospital, Lyon-Bron, France; ${ }^{6}$ Teaching Hospital of Rennes, Gynaecologic Department, University of Rennes, Rennes, France; ${ }^{7}$ Department of Obstetrics and Gynaecology, Hospital of Hautepierre, Strasbourg University Hospitals, Strasbourg, France; ${ }^{8}$ Department of Pathology, CHU Pontchaillou, Rennes, France; ${ }^{9}$ Department of Obstetrics and Gynaecology, Hôpital La Conception, APHM, Aix-Marseille University (AMU), Marseille, France; ${ }^{10} \mathrm{De}-$ partment of Gynaecologic and Oncologic Surgery, CHU Dijon, France; ${ }^{11}$ Pôle Mère-Femme, University Hospital Jean Minjoz, Besancon, France; and ${ }^{12}$ Pôle d'Obstétrique Reproduction Gynécologie, Centre Aliénor d'Aquitaine, Hôpital Pellegrin, Bordeaux, France

Reprint requests to: Xavier Carcopino, MD, PhD, Department of Obstetrics and Gynaecology Assistance Publique des Hôpitaux de Marseille (APHM) Hôpital Nord, Chemin des Bourrely, 13015 Marseille, France. E-mail: xcarco@free.fr

The authors have declared they have no conflicts of interest

The study protocol received institutional approval from the ethics committee of the French College of Obstetricians and Gynecologists, Paris, France (CEROG 2013-GYN-1001).

X.C. and L.P. were the project leaders. L.P., W.P., J.G., J.C., G.L., A.A., V.L., C.C., J.J.B., A.B., S.H., J.V., A.A., S.D., A.J., D.R., A.M., J.L.B., H.R and X.C. designed the study. L.P., J.G., J.C., G.L., A.A., V.L., C.C., J.J.B., A.B., S.H., J.V., A.A., S.D., A.J., D.R., A.M., J.L.B., H.R., and X.C. collected the data. J.M., W.P., L.P., J.G., and X.C. analyzed the data. L.P., W.P. J.G., J.M. and X.C. wrote the article. All authors approved the final manuscript. The corresponding and last authors had final responsibility for the decision to submit for publication.
$\mathrm{CI}=0.04-0.80, p=.024)$ as the achievement of negative endocervical margins $(\mathrm{aOR}=0.26,95 \% \mathrm{CI}=0.08-0.86, p=.027$ ).

Conclusions: Although the risk of post-LLETZ inadequate colposcopy is increased in patients with history of excisional therapy and with the thickness of the excised specimen, it could be reduced with the use of colposcopic guidance and the achievement of negative endocervical margins.

Key Words: LLETZ, colposcopy, follow up, risk,

high grade intraepithelial lesion, stenosis

arge loop excision of the transformation zone (LLETZ) is a - routine procedure worldwide, because it is the first-line treatment of high-grade intraepithelial lesion (HSIL) of the cervix. Quality criteria for optimal LLETZ include the completeness of excision with the achievement of negative margins, while producing the minimal excised volume and depth of excision to minimize subsequent obstetrical and neonatal morbidity. ${ }^{1,2}$ Obtaining negative margins is important, because incomplete excision exposes women to a significant risk of posttreatment residual and/or recurrent disease, particularly when the lesion involves the endocervical canal. ${ }^{3,4}$ However, this risk remains higher to the general female population, even when negative margins are achieved. Women who had had a LLETZ remain therefore exposed to a 3 - to 4-fold increased risk of developing subsequent cervical cancer at least for 20 years. ${ }^{5-8}$ Thus, prolonged and careful postLLETZ follow-up is mandatory, whatsoever the margins status. For the last decade, the value of human papillomavirus testing has been demonstrated in this indication. Although a negative human papillomavirus test has now been admitted as the best test of cure for patients, colposcopy remains needed when this test is found to be positive. ${ }^{9-12}$ Although being the key examination in this indication, the accuracy of colposcopy performed after previous excisional therapy of HSIL is however questionable because the healing process might result in changes in the appearance of the transformation zone (TZ). However, the main limitation of post-LLETZ colposcopic examination is the possibility of inadequate colposcopy due to the inability to visualize the entire TZ. Known risk factors for inadequate colposcopy include age, severity of lesion, and estrogen status of the patient. ${ }^{13}$ However, inadequate colposcopy is also one of the main adverse effects of excisional therapies of the cervix, including LLETZ. ${ }^{13}$ However, data on the precise risk factors for inadequate colposcopy after LLETZ are limited because most studies have focused on the sole risk of cervical stenosis without considering the position and visibility of the squamocolumnar junction. ${ }^{14-17}$ This point is however crucial because it is clinically essential to identify how post-LLETZ inadequate colposcopy could be avoided, thus preserving the possibility for the follow-up of these women. 
The aim of this study was to identify the risk factors of inadequate colposcopy after LLETZ. We also aimed to identify the risk factors for post-LLETZ cervical stenosis.

\section{MATERIALS AND METHODS}

This study is a secondary follow-up analysis of the ICORAD prospective multicenter observational study, performed in nine teaching hospitals in France, that aimed to investigate the benefit of the use of direct colposcopic vision for the guidance of LLETZ. ${ }^{18}$ ICORAD is the French acronym of "Impact de la Colposcopie sur la Résection à l'Anse Diathermique," which translates to "Impact of Colposcopy on LLETZ." From December 2013 to July 2014, a total of 249 all patients who had a LLETZ performed for the treatment of colposcopically and histologically proven HSIL were recruited to participate in this study. Only patients having a pretherapeutic fully visible cervical squamocolumnar junction were included; women with inadequate pretherapeutic colposcopy were not included in the current study. Written informed consents were obtained from all participants before excisional therapy was performed. Histological analysis of the LLETZ specimen showed invasive carcinoma in 13 cases and the absence of any intraepithelial lesion in 20 cases; all of these 33 cases were secondarily excluded from the study. Thus, a total of 216 patients were finally included. The study received approval from the ethics committee of the French College of Obstetricians and Gynecologists, Paris, France, in October 2013 (CEROG 2013-GYN-1001). Patients' characteristics such as age, parity, history of previous excisional procedure, indication for excision, and characteristics of the cervix were systematically recorded.

\section{Large Loop Excision of the Transformation Zone Procedures}

All procedures were performed under anesthesia, by a total of 45 gynecologists surgeons, according to their routine practice. Precise description of the characteristics of the surgeons was made in the initial ICORAD study publication. ${ }^{18}$ The use of colposcopy made during each LLETZ procedure was systematically documented. Three groups were considered: LLETZ achieved without any colposcopic examination at the time of the procedure and solely guided by the previous colposcopic report, LLETZ achieved under naked eyes vision immediately after a colposcopic examination had been performed, and LLETZ fully achieved under direct colposcopic vision (DCV). All LLETZ procedures were performed using a semicircular loop only. Dimensions of the loops were left to the appreciation of gynecologists, depending on the size of the cervix and of the type and size of the abnormal TZ. The abnormal $\mathrm{TZ}$ was considered to be large if at least three quadrants of the cervix were involved. The possible use of additional destructive therapy, using either ball diathermy or laser ablation, was systematically recorded. Specific attention was also given to how local hemostasis was achieved immediately after LLETZ. Thus, the use and exact duration of electrocauterization, cervical stitches, hemostatic compress, or vaginal clothing were systematically recorded. Finally, the occurrence of intraoperative complications was registered if any.

\section{Dimensions and Volume of LLETZ Specimens}

Gynecologists systematically measured exact dimensions and volume of opened LLETZ specimens at the time of procedure, just before formaldehyde fixation as previously reported. Before the study began, all gynecologists participating in the study received specific training regarding how the specimens' dimensions and volume were to be measured. Briefly, the volume was measured using the displacement fluid technique using a $25-\mathrm{mL}$ graduated tube. Dimensions recorded were the thickness, the length, and the circumference of each specimen, as illustrated in Figure 1. In case of multiple specimens were obtained, all the fragments were placed at the same time in the $25-\mathrm{mL}$ graduated tube for global volume assessment. In addition, although the assessment of the circumference of the excised tissue resulted in the addition of the circumference of each fragment, only the length and thickness from the largest fragment were considered.

\section{Follow-up Colposcopic Assessment}

All participants were invited to have a systematic follow-up colposcopy 3 to 6 months after LLETZ. Evaluation of the TZ was performed according to the classification of the Internal

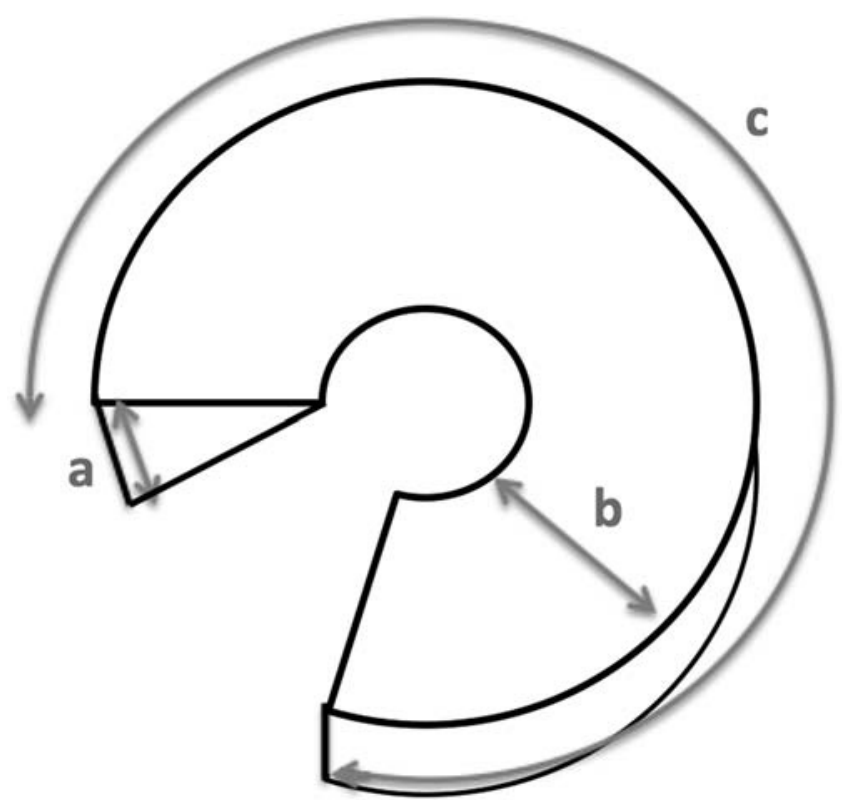

FIGURE 1. Drawing of an idealized opened excised specimen, with the dimensions used to determine thickness, length, and circumference (a, thickness; b, length; and c, circumference). 
Federation of Cervical Pathology and Colposcopy. ${ }^{19}$ Briefly, the $\mathrm{TZ}$ was classified as type 1 when it was entirely ectocervical and visible. Type 2 and 3 TZ both extend into the cervical canal, but whereas $\mathrm{TZ}$ was referred as a type 2 if the border between squamous and columnar epithelium was completely visible, it was referred as a type 3 if not. Primary end point of the study was the diagnosis of post-LLETZ inadequate colposcopy, defined by a type $3 \mathrm{TZ}$ and/or a cervical stenosis, resulting in a not fully visible cervical squamocolumnar junction. ${ }^{19,20}$ Secondary end point of the study was the post-LLETZ diagnosis cervical stenosis.

\section{Statistical Analysis}

Data were presented as the mean plus the standard error for continuous variables and as counts (percentages) for categorical data. Analysis of variance and Student $t$ tests were used to compare continuous data, and $\chi^{2}$ tests were used to compare categorical data. A binary logistic regression model was used to study the factors independently associated with posttreatment inadequate colposcopy and cervical stenosis. Only variables associated with a $\mathrm{p}$ value of less than .10 in univariate analysis were considered for inclusion in the multivariate model. Circumference and length of the excised specimen were removed because they strongly correlated to thickness of the specimen and were not significant after accounting for it. In addition, the use of local analgesia was also not included in the multivariate model because it was strongly associated with the use of DCV for the guidance of LLETZ. Statistical analyses were performed with IBM SPSS Statistics 20.0 (IBM Inc, New York, NY). A two-sided $p$ value of less than .05 was considered statistically significant.

\section{RESULTS}

Among the 216 patients included in the study, 59 (27.3\%) did not attend to the follow-up colposcopic assessment they were given 3 to 6 months after LLETZ therapy and were secondarily excluded. No significant difference between characteristics of patients who received follow-up compared with those who did not was observed. Finally, a total of 157 women were included in this study.

Patients' characteristics are summarized in Table 1. Colposcopic examinations were performed in a mean (SD) delay of 4.5 (2.9) months after LLETZ. Colposcopy was documented as inadequate in 22 cases (14\%). Women having post-LLETZ inadequate colposcopy were significantly older $(p=.015)$ and significantly less likely to be nulliparous ( $p=.049)$. Although the volume, circumference, and length of the specimens were not associated with a significant change in the probability of inadequate post-LLETZ colposcopic evaluation, the thickness of the specimens was found to significantly increase that risk. Thus, mean (SD) thickness of patients with inadequate post-LLETZ colposcopy were significantly more important than others $[10.5$ (4.7) $\mathrm{mm}$ vs 7.8 (3.3) mm, respectively, $p=.005]$. The best prediction for inadequate post-LLETZ colposcopic examination was found for a $9.5-\mathrm{mm}$ thickness cut-off (63.6\% sensitivity and $66.7 \%$ specificity). Although the final histological diagnosis and achievement of clear margins were not statistically different between patients having inadequate colposcopy and others, patients with inadequate colposcopy were significantly less likely to have negative endocervical margins [13 $(61.9 \%)$ vs $13113(84.3 \%)$, respectively, $p=.03]$. Finally, the use of colposcopy for the guidance of LLETZ was significantly associated with a reduced risk of inadequate colposcopy. Thus, compared with patients who had had a LLETZ performed without any use of colposcopy or just after colposcopic examination, the lowest proportion of inadequate colposcopy was observed among patients who had had LLETZ fully performed under DCV [19 (86.4\%) vs $3(13.6 \%)$, respectively, $p=.005]$.

\section{Factors Independently Associated With Post-LLETZ Inadequate Colposcopy}

In multivariate analysis, neither the age nor the nulliparity was found to have a significant impact on the probability of post-LLETZ inadequate colposcopic examination (see Table 2). Factors found to significantly increase the probability of postLLETZ inadequate colposcopy were a history of previous excisional cervical therapy [adjusted odds ratio $(\mathrm{aOR})=4.29,95 \%$ $\mathrm{CI}=1.12-16.37, p=.033]$ and the thickness of the specimen $(\mathrm{aOR}=3.12,95 \% \mathrm{CI}=1.02-9.60, p=.047)$. The use of DCV for the guidance of LLETZ was statistically associated with a decrease in the risk of posttreatment inadequate colposcopy $(\mathrm{aOR}=0.19,95 \% \mathrm{CI}=0.04-0.80, p=.024)$. Finally, the achievement of negative endocervical margins was found to significantly reduce the probability of post-LLETZ inadequate colposcopy $(\mathrm{aOR}=0.26,95 \% \mathrm{CI}=0.08-0.86, p=.027)$.

\section{Risk Factors for Post-LLETZ Cervical Stenosis}

A post-LLETZ cervical stenosis was diagnosed in five cases (3.2\%), representing $22.7 \%$ of the cases of inadequate colposcopic examinations (see Table 3). Compared with others, patients diagnosed with post-LLETZ cervical stenosis were significantly older $(p=.008)$. In addition, the thickness of the specimens was associated with a significant change in the probability of postLLETZ cervical stenosis. Thus, mean (SD) thickness of specimen from patients with post-LLETZ stenosis was significantly more important than others [12.8 (5.1) mm vs 8 (3.5) mm, respectively, $p=.004]$. Finally, patients with post-LLETZ stenosis had a higher mean volume of cervix excised. However, the difference was not significant [3.4 (0.5) $\mathrm{mL}$ vs $2.2(1.3) \mathrm{mL}$, respectively, $p=.057]$. In multivariate analysis, both the age of patients and the thickness of specimens were found to have a significant impact on the risk of post-LLETZ cervical stenosis $(\mathrm{aOR}=1.11$, $95 \% \mathrm{CI}=1.01-0.22, p=.029$, and $\mathrm{aOR}=1.21,95 \% \mathrm{CI}=$ $1.03-0.1 .42, p=.023$, respectively) (see Table 4).

\section{DISCUSSION}

\section{Main Findings}

With a proportion of $14 \%$ of patients diagnosed with inadequate post-LLETZ colposcopic examination, this study confirms that patients who had a LLETZ for the therapy of HSIL are exposed to a genuine risk of inadequate colposcopy, thus compromising follow-up. According to results from previous study, our results also show the risk of post-LLETZ inadequate colposcopy to depend on the thickness of the excised specimen. ${ }^{16}$ However, this study is the first to report this finding after LLETZ because it has been previously solely shown in patients who had had a laser conization. In addition, this study is the first to report this risk to depend on the use of DCV for the guidance of LLETZ and on the achievement of negative endocervical margins. Finally, this study also shows inadequate post-LLETZ colposcopy to partially result from cervical stenosis, with this risk depending on the age of patients and thickness of the excised specimen.

\section{Strength and Limitations}

Although the prospective nature of this study brings the possibility for optimal evaluation of the impact of the volume and dimensions of LLETZ specimen on the risk of further inadequate colposcopic examination, there are possible biases that need to be carefully considered when interpreting our results. In particular, colposcopic examination is known to be subjective, so is the characterization of the precise TZ type and the diagnosis of inadequate examination. We believe this point 
TABLE 1. Patients' Characteristics According to the Diagnostic of Post-LLETZ Inadequate Colposcopy

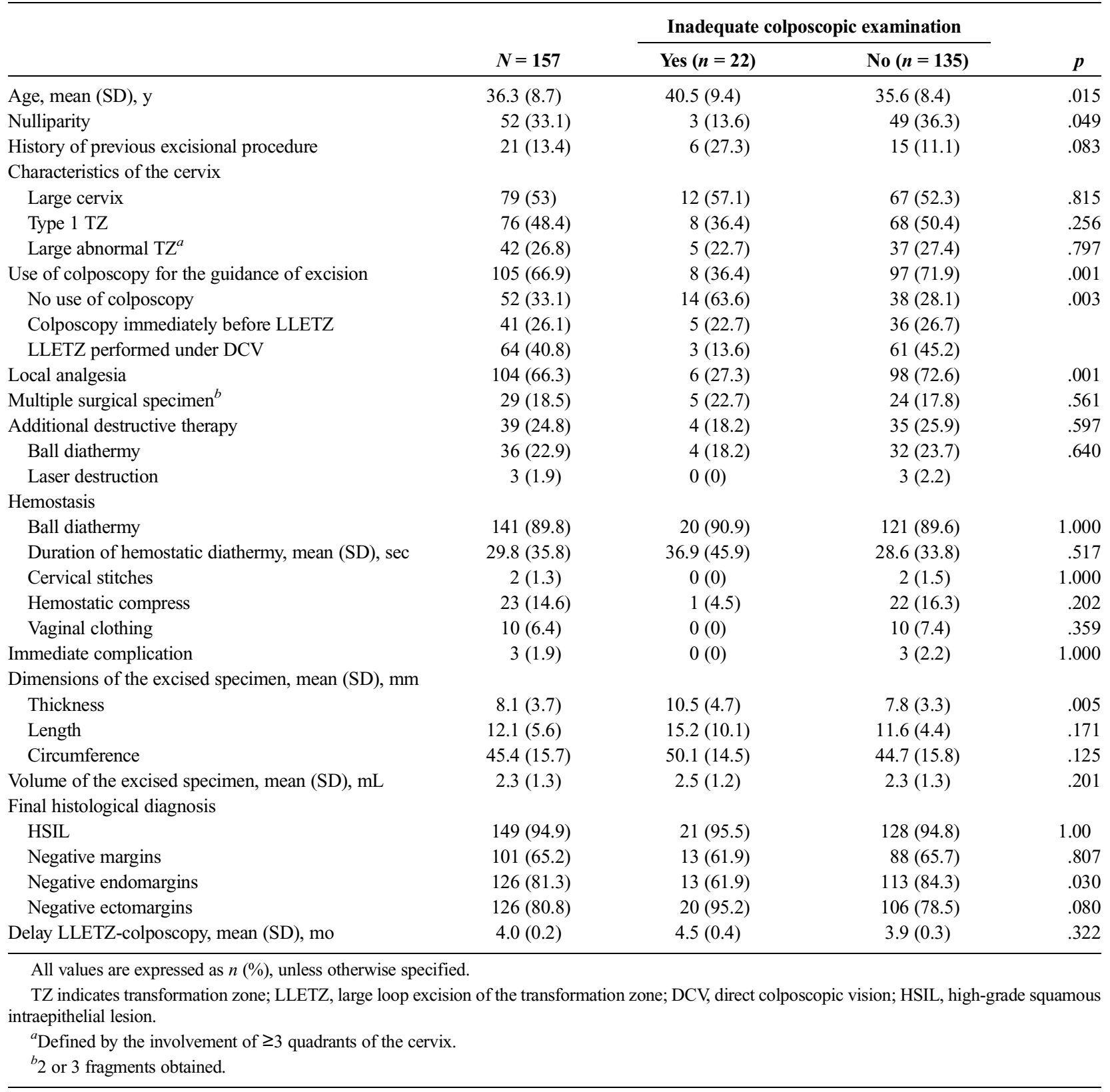

to be important because the range of inadequate colposcopic examination in literature varies from $3 \%$ to $20 \%$ depending on the population studied and the colposcopist. ${ }^{13,21,22}$ One should therefore consider the lack for additional post-LLETZ colposcopic examination by a second examiner as a limitation in the interpretation of our results. It is however noticeable that with a proportion of $14 \%$ post-LLETZ inadequate colposcopy, our results are consistent with those from previous literature that reported this risk to range from $6 \%$ to $30 \%{ }^{23,24}$ Because this diagnosis is far less subjective, we also aimed to identify the cases of postLLETZ stenosis. However, the small size of our study combined with the low proportion of cases of post-LLETZ cervical stenosis limits the interpretation of our results. Another point to be considered is that estimating the circumference of fragmented specimens by summing the circumference of the fragments could
TABLE 2. Factors Associated With the Occurrence of Post-LLETZ Unsatisfactory Colposcopic Examination (Binary Logistic Regression Model, $n=155$ )

\begin{tabular}{lll}
\hline & aOR (95\% CI) & $\boldsymbol{p}$ \\
\hline Age (per 1-y increase) & $1.04(0.98-1.11)$ & .180 \\
Nulliparity & $0.36(0.09-1.48)$ & .154 \\
History of previous excisional therapy & $4.29(1.12-16.37)$ & .033 \\
Use of direct colposcopic vision & $0.19(0.04-0.80)$ & .024 \\
Thickness of the excised specimen $\geq 10 \mathrm{~mm}$ & $3.12(1.02-9.60)$ & .047 \\
Negative endocervical margins & $0.26(0.08-0.86)$ & .027 \\
\hline \multicolumn{1}{c}{ aOR indicates adjusted odds ratio. } & & \\
\hline
\end{tabular}


TABLE 3. Patients' Characteristics According to the Diagnostic of Post-LLETZ Stenosis of the Cervical Os

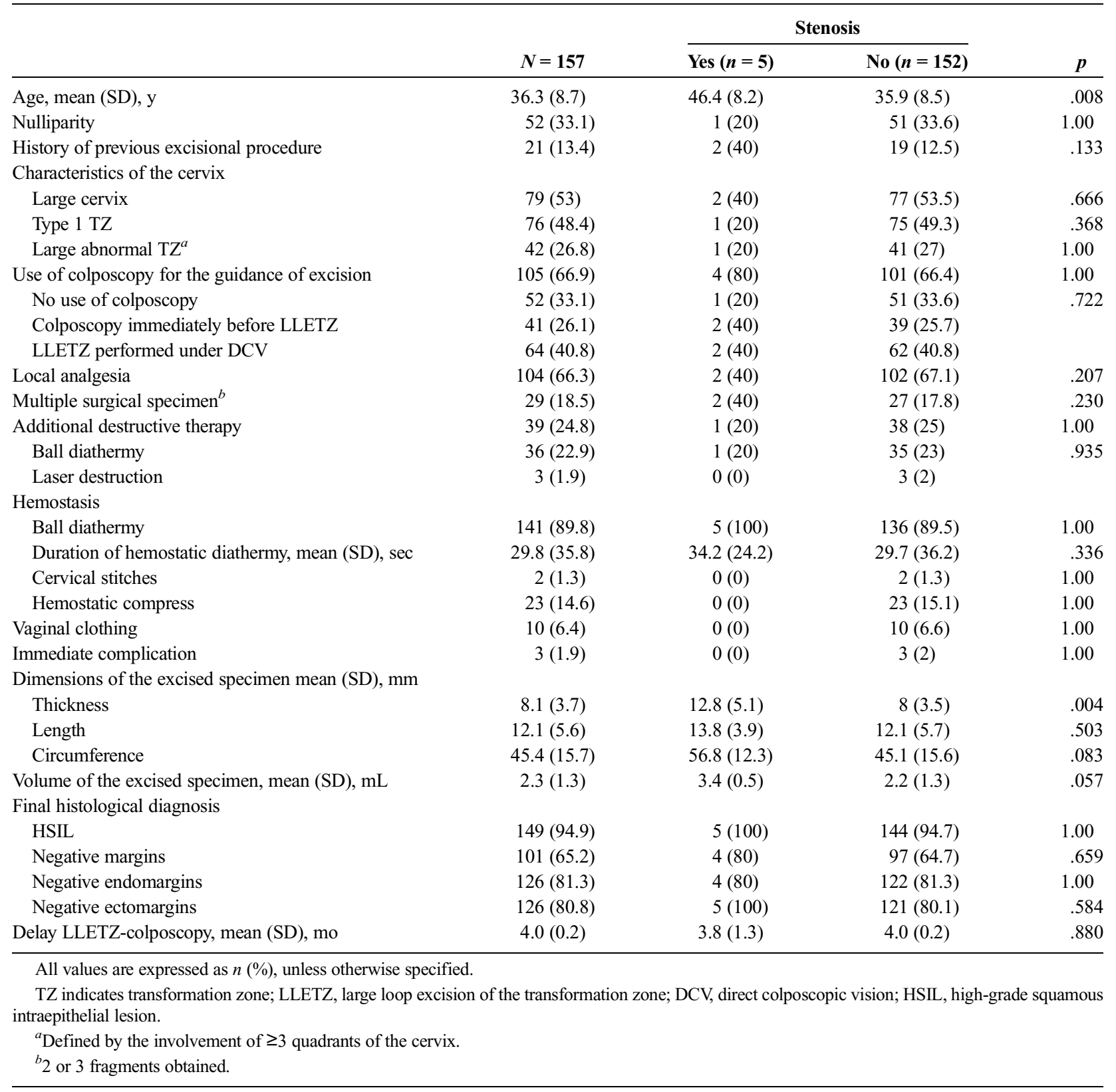

significantly have overestimated the circumference of the original specimen. However, multiple fragments were obtained in few cases $(18.5 \%)$, with no impact on the risk of inadequate post-LLETZ colposcopy (see Table 1). Even if a bias cannot be excluded, we believe the impact to be minimal. Finally, one should also consider that no sample size calculation was made because this study is a secondary follow-up analysis of a prospective project. ${ }^{18}$

\section{Interpretation}

In addition to the potential obstetrical impact and consequences on subsequent pregnancies, our results highlight the importance of the risk of inadequate colposcopic examination after LLETZ therapy. Although crucial, this point is however often under considered when making the decision of excisional therapy. In case when excisional therapy is considered, our results suggest to carefully put in balance the risk of compromised

TABLE 4. Factors Associated With Post-LLETZ Stenosis of the Cervical oz (Binary Logistic Regression Model, $N=157$ )

\begin{tabular}{llc}
\hline & aOR (95\% CI) & $\boldsymbol{p}$ \\
\hline Age (per 1-y increase) & $1.11(1.01-1.22)$ & .029 \\
$\begin{array}{l}\text { Thickness of the specimen } \\
\text { (per 1-mm increase) }\end{array}$ & $1.21(1.03-1.42)$ & .023 \\
\hline \multicolumn{2}{c}{ aOR indicates adjusted odds ratio. } & \\
\hline
\end{tabular}


follow-up due to inadequate colposcopic examination with the benefits of LLETZ. Interestingly, as previously reported, our results demonstrate the age of patients as a key factor for the risk of post-LLETZ inadequate colposcopic examination due to cervical stenosis. ${ }^{16}$ This finding is consistent with those from previous studies, and it is noticeable that in the absence of any excisional therapy, age itself has already been identified as a determinant factor in the position and visibility of the TZ. ${ }^{13} \mathrm{Al}-$ though, compared with other identified risk factors, the impact of the age seems to be minimal, this finding should be carefully considered when making the decision for excisional therapy in older patients. The two other factors that were identified to significantly increase the risk of inadequate post-LLETZ colposcopy were a history of excisional therapy and the thickness of the specimen excised. Considering those two factors, they both suggest the depth of excision to play a key role in the risk of subsequent inadequate colposcopic examination. Both the volume and the depth of the excision have been demonstrated to significantly impact the risk of subsequent obstetrical morbidity, with the risk of premature delivery being directly related to these two parameters. ${ }^{1,2}$ The depth and volume of excision are also likely to induce cervical morphological changes such as a reduction in the length of the cervix after LLETZ. ${ }^{25}$ Although one could hypothesize that the volume excised would significantly impact the risk of inadequate post-LLETZ colposcopic examination, our results do not support this hypothesis. Thus, although the volume directly depends on the thickness of the excision performed, it also depends on all the other dimensions of the excised specimen. However, because the length and the circumference of the excised specimen were not found to increase the risk of inadequate post-LLETZ colposcopic examination, our results suggest that a large but not deep excision is not likely to end in making the post-LLETZ colposcopic follow-up impossible. This result therefore brings additional argument to minimize the thickness of the excised specimen, ideally in precisely adapting the LLETZ to the precise characteristics of the abnormal $\mathrm{TZ}$ and to the exact position of the squamocolumnar junction.

It is important to note that the use of colposcopy during procedures was left to the appreciation of surgeons according to their routine practice, and our study therefore illustrates the wide range of variations in how LLETZ is performed. In previous studies, the use of DCV was shown to help in minimizing the dimensions of the excised specimen, including the thickness, without compromising the margins status. ${ }^{18,26,27}$ In addition to the potential subsequent obstetrical benefit, the systematic use of DCV should therefore also be recommended to reduce the risk of compromised post-LLETZ follow-up as a result of the reduction of the thickness of the excised tissue. However, independently to the thickness of the excised specimen, our study shows the use of DCV to significantly and massively reduce the risk of inadequate colposcopic examination. It is noticeable that this effect was not observed when solely considering the risk of post-LLETZ cervical stenosis. Although this finding suggests that guidance of LLETZ by DCV mostly ends in a reduction of the probability of a type $3 \mathrm{TZ}$, we cannot exclude the possibility that colposcopists from this group were more likely to identify the squamocolumnar junction, resulting in a lower proportion of type $3 \mathrm{TZs}$. At this stage, we acknowledge that this result should be considered with caution and that other prospective studies with double post-LLETZ colposcopic assessment are needed to properly evaluate this parameter.

Finally, we found the achievement of negative margins to significantly, independently, and strongly reduce the risk of post-LLETZ inadequate colposcopic evaluation. It is noticeable that this finding was not observed for the ectocervical margins.
Although we feel difficult to properly explain this result, it might suggest that when not achieving clear endocervical margins and therefore not fully excising the squamocolumnar junction, the healing process may result in the burying of the junction deep inside the cervical canal. We acknowledge this finding to be surprising and that more prospective studies are needed to properly investigate this parameter. At this stage, we believe our results to provide genuine arguments to promote that all efforts should be made to achieve ideal balance between minimal excision and negative margins. This point seems crucial, not only to minimize the risk of subsequent obstetrical morbidity but also to minimize the risk of compromised follow-up in women with identified increased risk to develop recurrence and subsequent invasive cancer.

\section{CONCLUSIONS}

After LLETZ, patients with initial type 1 or $2 \mathrm{TZ}$ are exposed to the risk of inadequate colposcopic examination, thus compromising follow-up. This risk partially results from cervical stenosis, mostly depending on the age of patients, and should be put in balance when making the decision for excisional therapy, particularly in older women. With this risk depending on the thickness of the excised specimen as well as on the use of DCV for the guidance of LLETZ and on the achievement of negative endocervical margins, this study brings additional arguments to recommend the systematic use of DCV for the guidance of LLETZ and optimal excision. More prospective studies remain needed to confirm our findings.

\section{ACKNOWLEDGMENTS}

The authors thank all the women who participated in this study.

\section{REFERENCES}

1. Kyrgiou M, Koliopoulos G, Martin-Hirsch P, et al. Obstetric outcomes after conservative treatment for intraepithelial or early invasive cervical lesions: systematic review and meta-analysis. Lancet 2006;367:489-98.

2. Kyrgiou M, Athanasiou A, Paraskevaidi M, et al. Adverse obstetric outcomes after local treatment for cervical preinvasive and early invasive disease according to cone depth: systematic review and meta-analysis. BMJ 2016;354:13633.

3. Ghaem-Maghami S, De-Silva D, Tipples M, et al. Determinants of success in treating cervical intraepithelial neoplasia. BJOG 2011;118:679-84.

4. Ghaem-Maghami S, Sagi S, Majeed G, et al. Incomplete excision of cervical intraepithelial neoplasia and risk of treatment failure: a meta-analysis. Lancet Oncol 2007;8:985-93.

5. Rebolj M, Helmerhorst T, Habbema D, et al. Risk of cervical cancer after completed post-treatment follow-up of cervical intraepithelial neoplasia: population based cohort study. BMJ 2012;345:e6855.

6. Strander B, Andersson-Ellström A, Milsom I, et al. Long term risk of invasive cancer after treatment for cervical intraepithelial neoplasia grade 3: population based cohort study. BMJ 2007;335:1077.

7. Soutter WP, de Barros Lopes A, Fletcher A, et al. Invasive cervical cancer after conservative therapy for cervical intraepithelial neoplasia. Lancet Lond Engl 1997;349:978-80.

8. Melnikow J, McGahan C, Sawaya GF, et al. Cervical intraepithelial neoplasia outcomes after treatment: long-term follow-up from the British Columbia Cohort Study. J Natl Cancer Inst 2009;101:721-8.

9. Verguts J, Bronselaer B, Donders G, et al. Prediction of recurrence after treatment for high-grade cervical intraepithelial neoplasia: the role of human papillomavirus testing and age at conisation. BJOG 2006; 113:1303-7. 
10. Paraskevaidis E, Arbyn M, Sotiriadis A, et al. The role of HPV DNA testing in the follow-up period after treatment for CIN: a systematic review of the literature. Cancer Treat Rev 2004;30:205-11.

11. Kocken M, Uijterwaal MH, de Vries AL, et al. High-risk human papillomavirus testing versus cytology in predicting post-treatment disease in women treated for high-grade cervical disease: a systematic review and meta-analysis. Gynecol Oncol 2012;125: $500-7$

12. Arbyn M, Ronco G, Anttila A, et al. Evidence regarding human papillomavirus testing in secondary prevention of cervical cancer. Vaccine 2012;30(suppl 5):F88-99.

13. Carcopino X, Akkawi R, Conroy R, et al. Specific timing for colposcopy: is it worthwhile? Obstet Gynecol 2008;111(2 Pt 1):373-7.

14. Penna C, Fambrini M, Fallani MG, et al. Laser CO2 conization in postmenopausal age: risk of cervical stenosis and unsatisfactory follow-up. Gynecol Oncol 2005;96:771-5.

15. Baldauf JJ, Dreyfus M, Ritter J, et al. Risk of cervical stenosis after large loop excision or laser conization. Obstet Gynecol 1996;88: 933-8

16. Houlard S, Perrotin F, Fourquet F, et al. Risk factors for cervical stenosis after laser cone biopsy. Eur J Obstet Gynecol Reprod Biol 2002;104: 144-7.

17. Kliemann LM, Silva M, Reinheimer M, et al. Minimal cold knife conization height for high-grade cervical squamous intraepithelial lesion treatment. Eur J Obstet Gynecol Reprod Biol 2012;165:342-6.

18. Preaubert L, Gondry J, Mancini J, et al. Benefits of direct colposcopic vision for optimal LLETZ procedure: a prospective multicenter study. J Low Genit Tract Dis 2016;20:15-21.
19. Bornstein J, Bentley J, Bösze P, et al. 2011 colposcopic terminology of the International Federation for Cervical Pathology and Colposcopy. Obstet Gynecol 2012;120:166-72.

20. Tatti S, Bornstein J, Prendiville W. Colposcopy: a global perspective: introduction of the new IFCPC colposcopy terminology. Obstet Gynecol Clin North Am 2013;40:235-50.

21. Makkar B, Batra S, Gandhi G, et al. Vaginal misoprostol versus vaginal estradiol in overcoming unsatisfactory colposcopy. Gynecol Obstet Invest 2014;77:176-9.

22. Pretorius RG, Belinson JL, Peterson P, et al. Which colposcopies should include endocervical curettage? J Low Genit Tract Dis 2015; 19:278-81.

23. Thompson V, Marin R. Is colposcopy necessary at twelve months after large loop excision of the transformation zone? A clinical audit. Aust N Z J Obstet Gynaecol 2013;53:571-3.

24. Suh-Burgmann EJ, Whall-Strojwas D, Chang Y, et al. Risk factors for cervical stenosis after loop electrocautery excision procedure. Obstet Gynecol 2000;96(5 Pt 1):657-60.

25. Carcopino X, Maycock JA, Mancini J, et al. Image assessment of cervical dimensions after LLETZ: a prospective observational study. BJOG Int J Obstet Gynaecol 2013;120:472-8.

26. Grisot C, Mancini J, Giusiano S, d'Ercole C, et al. How to optimize excisional procedures for the treatment of CIN? The role of colposcopy. Arch Gynecol Obstet 2012;285:1383-90.

27. Carcopino X, Mancini J, Charpin C, et al. Direct colposcopic vision used with the LLETZ procedure for optimal treatment of CIN: results of joint cohort studies. Arch Gynecol Obstet 2013;288: 1087-94 